\title{
CRIM-negative infantile Pompe disease: characterization of immune responses in patients treated with ERT monotherapy
}

\author{
Kathryn L. Berrier, MS, CGC1',Zoheb B. Kazi, MBBS'1, Sean N. Prater, MD, MRes', Deeksha S. Bali, PhD', \\ Jennifer Goldstein, PhD, CGC1, Mihaela C. Stefanescu, BS', Catherine W. Rehder, PhD², \\ Eleanor G. Botha, MS, CGC 3 , Carolyn Ellaway, MBBS, PhD ${ }^{4}$, Kaustuv Bhattacharya, MD', Anna \\ Tylki-Szymanska, MD, PhD ${ }^{5}$, Nesrin Karabul, MD' ${ }^{6}$ Amy S. Rosenburg, MD and Priya S. Kishnani, MD ${ }^{1}$
}

\begin{abstract}
Purpose: Enzyme replacement therapy (ERT) with recombinant human acid $\alpha$-glucosidase (rhGAA) prolongs survival in infantile Pompe disease (IPD). However, the majority of cross-reactive immunologic material (CRIM)-negative $(\mathrm{CN})$ patients have immune responses with significant clinical decline despite continued ERT. We aimed to characterize immune responses in CN patients with IPD receiving ERT monotherapy.
\end{abstract}

Methods: A chart review identified $20 \mathrm{CN}$ patients with IPD treated with ERT monotherapy for $\geq 6$ months. Patients were stratified by anti-rhGAA antibody titers: high sustained antibody titers (HSAT; $\geq 51,200$ ) at least twice; low titers (LT; $<6,400$ ) throughout treatment; or sustained intermediate titers (SIT; 6,400-25,600).

Results: Despite early initiation of treatment, the majority (85\%) of $\mathrm{CN}$ patients developed significant antibody titers, most with HSAT associated with invasive ventilation and death. Nearly all patients with HSAT had at least one nonsense GAA mutation, whereas the LT group exclusively carried splice-site or frameshift mutations. Only one patient in the HSAT group is currently alive after successful immune modulation in the entrenched setting.

Conclusion: Immunological responses are a significant risk in $\mathrm{CN}$ IPD; thus induction of immune tolerance in the naive setting should strongly be considered. Further exploration of factors influencing immune responses is required, particularly with the advent of newborn screening for Pompe disease.

Genet Med advance online publication 5 March 2015

Key Words: alglucosidase alfa; antibodies; enzyme replacement therapy; immune tolerance; Pompe disease

\section{INTRODUCTION}

In Pompe disease (OMIM 232300; acid maltase deficiency, glycogen storage disease type II), a deficiency of lysosomal acid $\alpha$-glucosidase (GAA) leads to the accumulation of glycogen in skeletal, cardiac, and smooth muscle, resulting in progressive, debilitating muscle weakness. If left untreated, infants with the classic infantile form of Pompe disease (IPD) typically succumb to the disease by cardiopulmonary failure within the first 2 years of life. However, intravenous enzyme replacement therapy (ERT) with recombinant human GAA (rhGAA), or alglucosidase alfa (Lumizyme), has improved clinical outcomes and prolonged survival in patients with IPD.

Despite improved clinical outcomes, including prolonged overall and ventilator-free survival of patients with IPD, ${ }^{1-4}$ the efficacy of ERT is diminished for some patients who mount an immune response against rhGAA. Previous studies demonstrated that cross-reactive immunologic material (CRIM) status is highly correlated with clinical response to treatment. ${ }^{5,6}$
CRIM-positive (CP) patients generally respond well to ERT owing to the presence of residual GAA expression. On the other hand, patients with no residual protein expression are categorized as CRIM-negative $(\mathrm{CN})$. In general, when compared with $\mathrm{CP}$ patients who tolerize to ERT over time, CN patients mount an immune response against rhGAA, resulting in significant clinical decline and ultimately ventilator dependence or death by 27.1 months, despite the continuation of ERT. ${ }^{5}$

Physicians caring for patients with IPD treated with a therapeutic protein such as rhGAA are thus charged with the challenge of also managing significant immune responses. Our group has demonstrated the success of immune tolerance induction (ITI) protocols along with ERT in both the naive setting, to prevent the development of high antibody titers, ${ }^{7-9}$ and the entrenched setting, to suppress an immune response once high antibody titers have already developed. ${ }^{6,10}$ However, ITI has proved to be most successful in the naive

The first two authors contributed equally to this work.

${ }^{1}$ Department of Pediatrics, Division of Medical Genetics, Duke University Medical Center, Durham, North Carolina, USA; ${ }^{2}$ Department of Pathology, Duke University Medical Center, Durham, North Carolina, USA; ${ }^{3}$ Department of Human Genetics, Emory University, Atlanta, Georgia, USA; ${ }^{4}$ Western Sydney Genetics Program, Children's Hospital at Westmead, Sydney, Australia; ${ }^{5}$ Department of Pediatrics, Nutrition and Metabolic Diseases, The Children's Memorial Health Institute, Warsaw, Poland; ${ }^{6}$ Center for Pediatric and Adolescent Medicine, University Medical Center, Mainz, Germany; ${ }^{7}$ Division of Therapeutic Proteins, Center for Drug Evaluation and Research, US Food and Drug Administration, Silver Spring, Maryland, USA. Correspondence: Priya S. Kishnani (priya.kishnani@duke.edu) 
setting, which requires a short 5-week regimen of immune modulation initiated at the start of ERT, compared with a significantly longer, multipronged approach for treating patients with an entrenched immune response to rhGAA. ${ }^{6,10}$

The current literature reflects how clinical outcomes of $\mathrm{CN}$ patients treated with ERT and ITI surpass those receiving ERT monotherapy. ${ }^{9}$ However, three cases have been reported in which $\mathrm{CN}$ patients have done relatively well clinically in the absence of high sustained antibody titers (HSAT) without the use of ITI. ${ }^{11,12}$ Yet, it is important to note that one of these patients received omalizumab, an IgG monoclonal antibody that binds IgE, for a severe allergic reaction to alglucosidase alfa infusion. ${ }^{11}$ The role of this drug in immunomodulation is yet to be reported.

Because the use of ITI protocols is becoming more prevalent, understanding the factors influencing immune responses in the CN IPD population is important; such knowledge is essential for guiding further implementation of ITI protocols. However, a fundamental understanding of the long-term clinical outcomes of CN patients with IPD treated with ERT alone (ERT monotherapy) is missing. In this study we aimed to retrospectively assess the immune responses in our cohort of $\mathrm{CN}$ patients with IPD receiving rhGAA, which we believe is the largest in the world. We did this to determine what proportion of $\mathrm{CN}$ patients develop significant immune responses, which negatively affect clinical outcomes, and to consider the influence of potentially modifying factors in the development of an immune response, such as GAA genotype or age at which ERT is initiated. This cohort represents an invaluable set of data and provides a unique opportunity to examine responses to ERT alone, especially as the treatment paradigm for CN patients with IPD continues to shift toward the implementation of ITI in the naive setting.

\section{MATERIALS AND METHODS}

This was a retrospective chart review of our large cohort of 183 patients with IPD, taken from our previously published cohort ${ }^{5}$ and enrolled in Duke Institutional Review Board-approved protocol 00001562 (LDN6709 Site 206; clinicaltrials.gov identifier NCT01665326), to determine CRIM status and provide longitudinal follow-up.Of those 183 patients, we identified 58 who were determined to be $\mathrm{CN}, 20$ of whom were treated with alglucosidase alfa ERT monotherapy for at least 6 months at cumulative doses of $20-40 \mathrm{mg} / \mathrm{kg}$ every 2 weeks. We included patients who had been on ITI regimens that were unsuccessful in preventing the development of HSAT. We also included one patient (patient 10; HSAT group) who was successfully treated with ITI in the entrenched setting; data were used until ITI was initiated at week 87. Patients were excluded from the data analysis if ITI was administered in the naive setting.

\section{Laboratory methods}

$\mathrm{CN}$ status was first determined based on the reactivity of a pool of monoclonal and/or polyclonal anti-rhGAA antibodies capable of recognizing both native and recombinant GAA protein bands, using western blot analysis on skin fibroblast cell extracts. If none of the GAA protein forms (unprocessed precursor band at $110 \mathrm{kDa}$ or processed forms bands at 95,76 , and $70 \mathrm{kDa}$ ) were detected, the patient is categorized as $\mathrm{CN} .{ }^{5}$ In one case $\mathrm{CN}$ status was determined using western blot analysis of amniocytes following the prior diagnosis of the proband sibling. ${ }^{12}$ For all patients, final CN status was assigned once the results of western blot analysis were correlated with GAA mutation genotype. ${ }^{13}$

\section{Anti-rhGAA IgG antibodies}

Anti-rhGAA IgG antibodies were ascertained using enzymelinked immunosorbent assays and confirmed using radioimmunoprecipitation, as described previously, ${ }^{2}$ and were assessed by Genzyme Corporation (Framingham, MA) at baseline and at regular intervals throughout treatment. CN patients with IPD were further divided into three groups based on antirhGAA antibody titer levels and the need for further clinical intervention ${ }^{6,14}$ :

1. HSAT group: anti-rhGAA $\operatorname{IgG}$ antibodies measured $\geq 51,200$ on two or more occasions after 6 months of ERT.

2. Low titer (LT) group: IgG antibodies remained $<6,400$ throughout the course of ERT.

3. Sustained intermediate titer (SIT) group: IgG antibodies ranged from 6,400 to 25,600 .

\section{Clinical parameters}

Baseline and follow-up data, including age at diagnosis, age at initiation of ERT, invasive ventilator-free survival, overall survival, and left ventricular mass index (LVMI), were obtained to assess treatment outcomes in these three groups.

\section{Statistical analysis}

Survival data were analyzed using the Kaplan-Meier method; two-tailed $P$ values were generated using the log-rank test. ${ }^{15}$ Analyses were performed with STATA version 13.1 (StataCorp LP, College Station, TX). Because of the small sample size, descriptive data are presented as medians.

\section{RESULTS}

Of our total IPD cohort $(n=183)$, established between 1999 and 1 February 2014, 58 were identified as CN, which to our knowledge represents the largest CN IPD cohort in the current literature. From this group, $23 \mathrm{CN}$ patients with IPD were excluded from analysis because ITI treatment was administered in the naive setting; for 15 other CN patients with IPD, death occurred before 6 months on ERT or there were insufficient follow-up data; thus the resulting data analysis included $20 \mathrm{CN}$ patients with IPD (Figure 1).

\section{Anti-rhGAA antibody titers}

The majority (85\%) of $\mathrm{CN}$ patients (17/20) developed clinically significant IgG antibody titers to rhGAA. Thirteen 
mounted HSAT, with median peak titers of 204,800 by 24 weeks on ERT (Table 1). Four patients had SIT, with peak titers of 25,600 and 51,200 . Three patients had LT peaking at $200(n=2)$ and $800(n=1)$.

\section{Spectrum of GAA mutations}

We identified GAA mutations in our cohort (Table 2) and assessed the types of mutations seen in each titer group

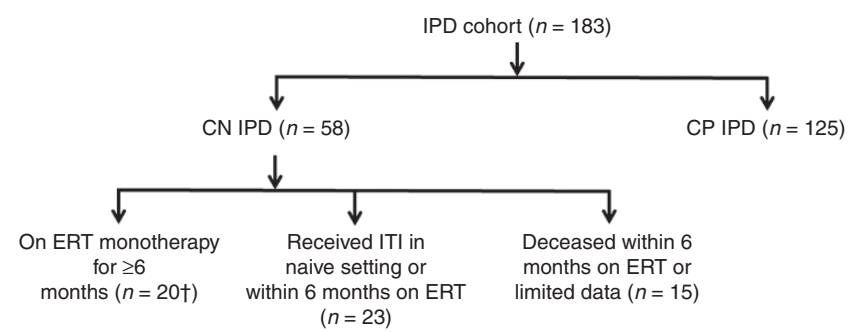

Figure 1 Cohort of patients with infantile Pompe disease (IPD). ${ }^{\dagger}$ One patient rescued with an immune tolerance induction (ITI) protocol in the entrenched setting after 6 months of enzyme replacement therapy (ERT); therefore, data for this patient were included up until the initiation of ITI. ${ }^{10}$ Two patients were included because ITI regimen was unsuccessful, with a persistence of antibody titers. ${ }^{19,20} \mathrm{CRIM}$, cross-reactive immunologic material (CRIM); CN, CRIM-negative; CP, CRIM-positive.
(Supplementary Figure S1 online). Ten of 13 patients in the HSAT group carried at least one nonsense mutation. The total frequency of nonsense mutations observed in the HSAT group was 18/26 total alleles (69\%); frameshift mutation allele frequency was $7 / 26$ alleles (27\%), and splice-site mutation allele frequency was $1 / 26$ alleles (4\%). Three of eight alleles (38\%) in the SIT group were frameshift mutations, two (25\%) were missense initiator codon mutations, two (25\%) were nonsense mutations, and one (13\%) was a splice-site mutation. Three of six alleles (50\%) observed in the LT group were splice-site mutations and three $(50 \%)$ were frameshift mutations. All patients were unrelated with the exception of one sibling pair (patient 14 of the SIT group and patient 18 of the LT group), who were compound heterozygotes for the c.546+2_+5delTGGG splice-site mutation and c.1650dupG frameshift mutation, as first reported by Al Khallaf et al. ${ }^{12}$ Unlike the SIT and HSAT groups, no patient in the LT group carried a nonsense mutation.

\section{Clinical parameters}

Table 3 provides demographic and clinical parameters for this international cohort of $\mathrm{CN}$ patients with IPD receiving ERT monotherapy. Four patients started ERT before 1 month of

Table 1 Antibody response in HSAT, SIT, and LT groups at peak titer and last titer measurements

\begin{tabular}{|c|c|c|c|c|c|}
\hline Group & Patient & Peak titers & $\begin{array}{l}\text { Weeks on ERT } \\
\text { at peak titers }\end{array}$ & Last titers & $\begin{array}{l}\text { Weeks on ERT } \\
\text { at last titers }\end{array}$ \\
\hline \multirow[t]{15}{*}{ HSAT } & 1 & 204,800 & 78 & 102,400 & 152 \\
\hline & 2 & 204,800 & 60 & 102,400 & 181 \\
\hline & 3 & 102,400 & 24 & 51,200 & 36 \\
\hline & 4 & 204,800 & 64 & 204,800 & 64 \\
\hline & 5 & 409,600 & 64 & 409,600 & 82 \\
\hline & 6 & $1,638,400$ & 52 & $1,638,400$ & 64 \\
\hline & 7 & 204,800 & 38 & 102,400 & 52 \\
\hline & 8 & 51,200 & 24 & 25,600 & 38 \\
\hline & 9 & 409,600 & 64 & 409,600 & 64 \\
\hline & 10 & 819,200 & 86 & $819,200^{\mathrm{a}}$ & 86 \\
\hline & 11 & 51,200 & 28 & 51,200 & 63 \\
\hline & 12 & 819,200 & 130 & 409,600 & 138 \\
\hline & 13 & 819,200 & 29 & 819,200 & 29 \\
\hline & Median & 204,800 & 60 & 204,800 & 64 \\
\hline & Range & $51,200-1,638,400$ & $24-130$ & $25,600-1,638,400$ & 29-181 \\
\hline \multirow[t]{6}{*}{ SIT } & 14 & 51,200 & 15 & 6,400 & 202 \\
\hline & 15 & 25,600 & 16 & 6,400 & 90 \\
\hline & 16 & 25,600 & 117 & 6,400 & 194 \\
\hline & 17 & 25,600 & 24 & 12,800 & 132 \\
\hline & Median & 25,600 & 20 & 6,400 & 145 \\
\hline & Range & $25,600-51,200$ & $15-117$ & $6,400-25,600$ & 90-202 \\
\hline \multirow[t]{5}{*}{ LTs } & 18 & 800 & 26 & 100 & 112 \\
\hline & 19 & 200 & 100 & 100 & 110 \\
\hline & 20 & 200 & 48 & 0 & 84 \\
\hline & Median & 200 & 48 & 100 & 110 \\
\hline & Range & $200-800$ & $26-100$ & $0-100$ & $84-112$ \\
\hline
\end{tabular}

ERT, enzyme replacement therapy; HSAT, high sustained antibody titers; LT, low titers; SIT, sustained intermediate titers.

andicates the last titer value before the initiation of immune tolerance induction. 

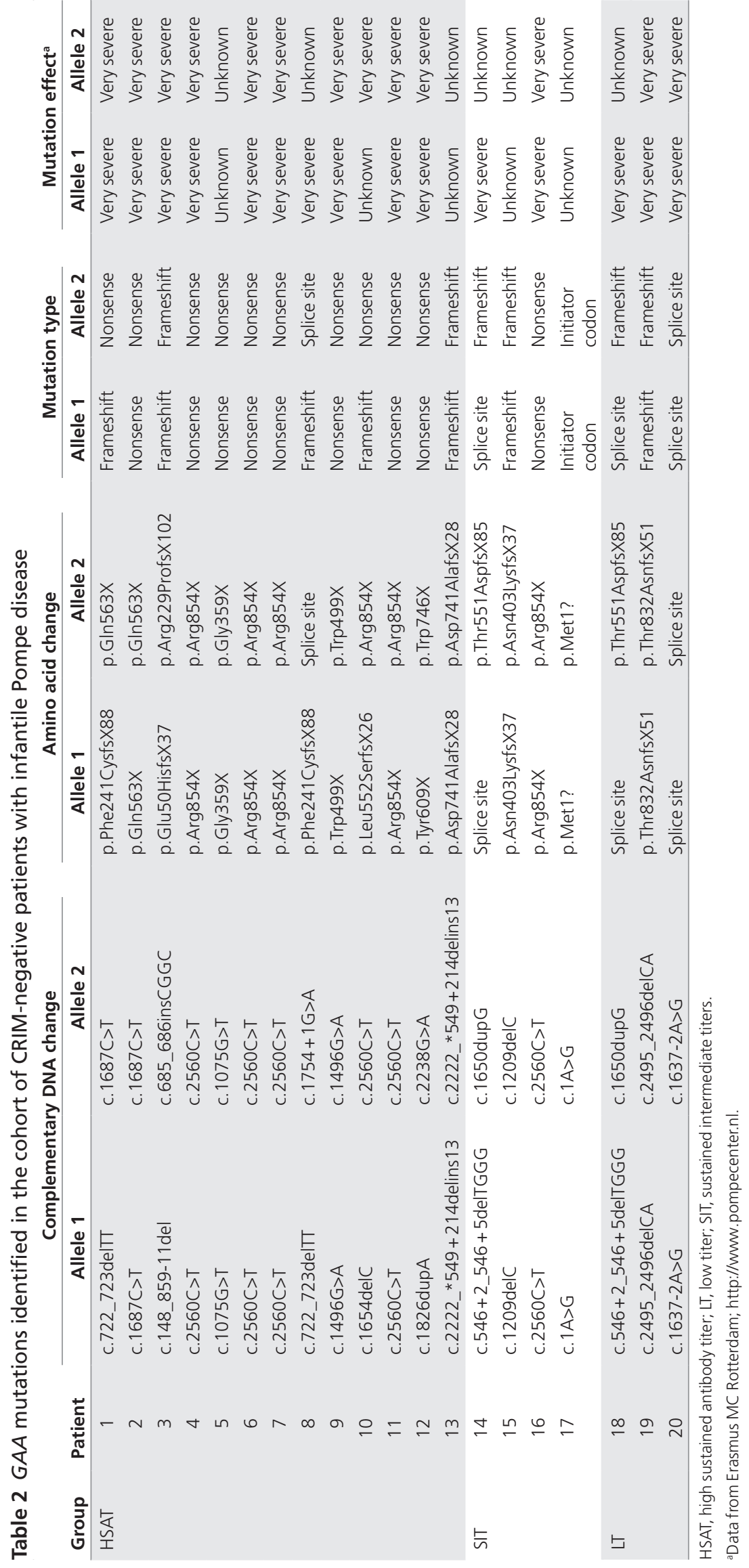
age, with variable clinical outcomes: two have not developed an immune response against rhGAA (patients 18 and 20), whereas two others responded poorly, with death at 18 and 45 months of age (patients 9 and 16). Thus it seems that early initiation of ERT does not necessarily prevent the development of an antibody response to ERT.

\section{Invasive ventilator-free and overall survival}

No patients required invasive ventilator support at baseline; however, $55 \%(n=11)$ required invasive ventilation by a median age of 13.8 months (range, 8.2-33.6 months) (Figure 2a). Four patients (20\%) died (median age at death, 23.1 months; range, 18.0-45.0 months) before requiring invasive ventilation, and the

Table 3 Demographic and clinical parameters of the cohort of CN patients with IPD

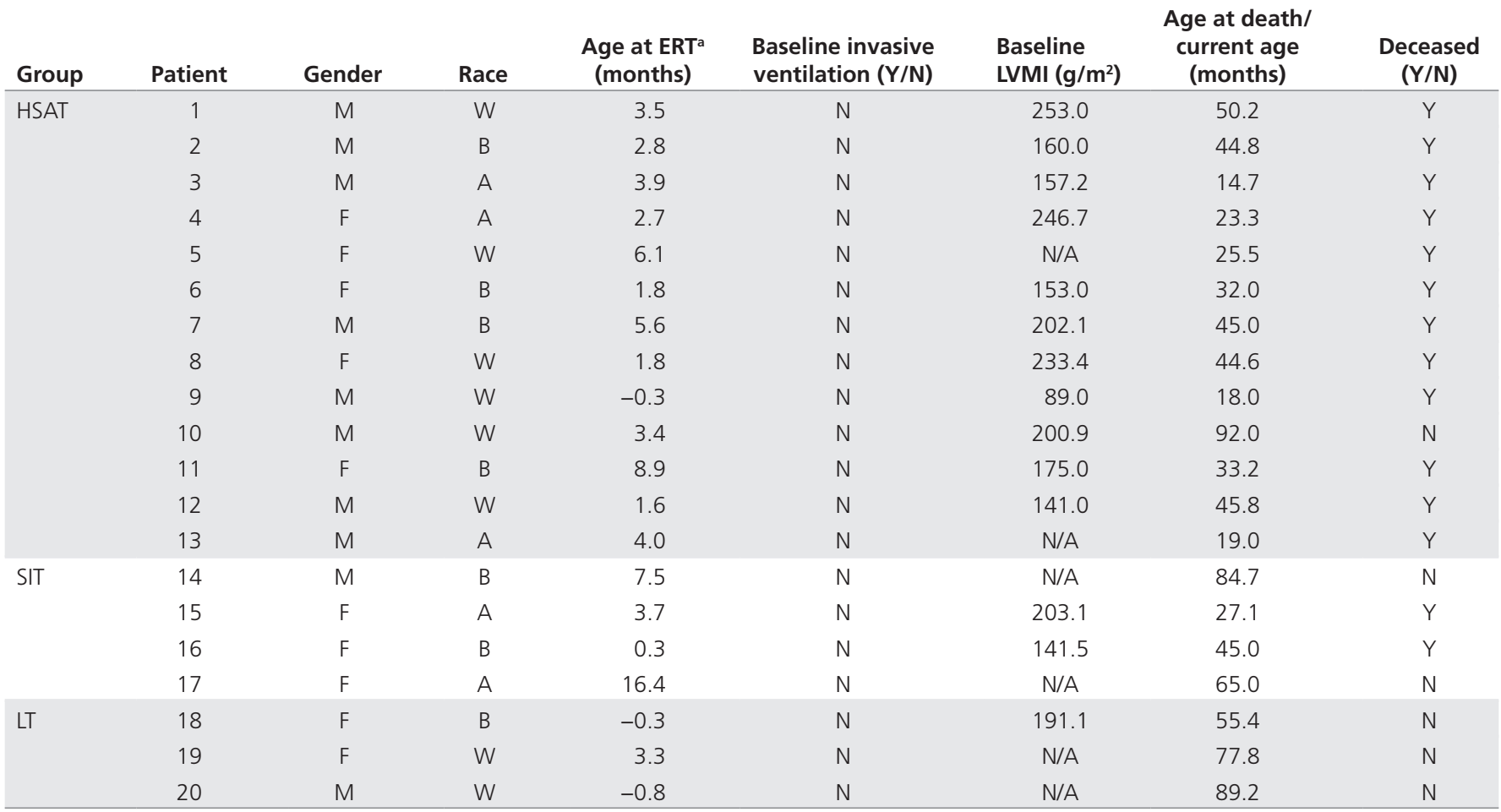

A, Asian; B, black; ERT, enzyme replacement therapy; F, female; HSAT, high sustained antibody titers; LT, low titers; LVMI, left ventricular mass index; M, male; N, no; N/A, not available; SIT, sustained intermediate titers; $W$, white; $Y$, yes.

age at ERT corrected by gestational age.

a

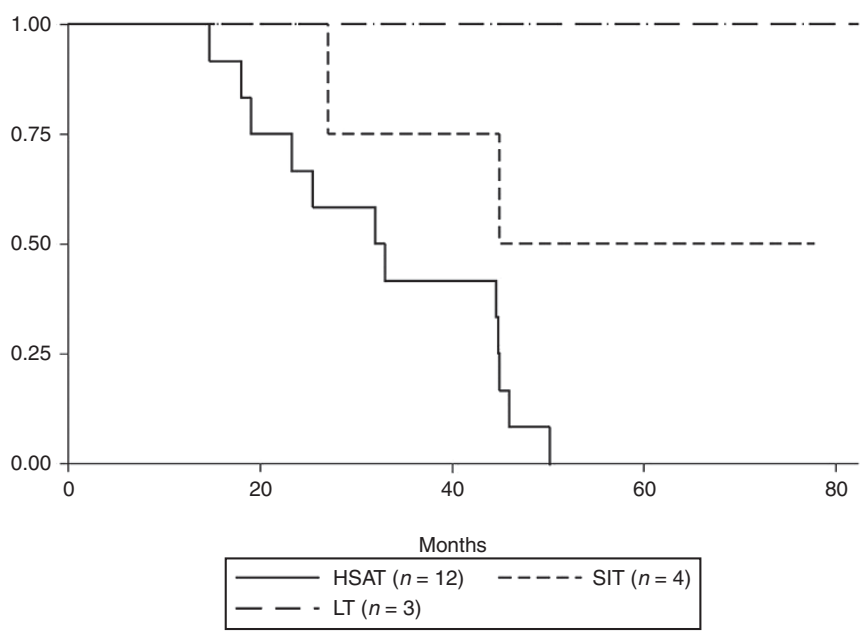

b

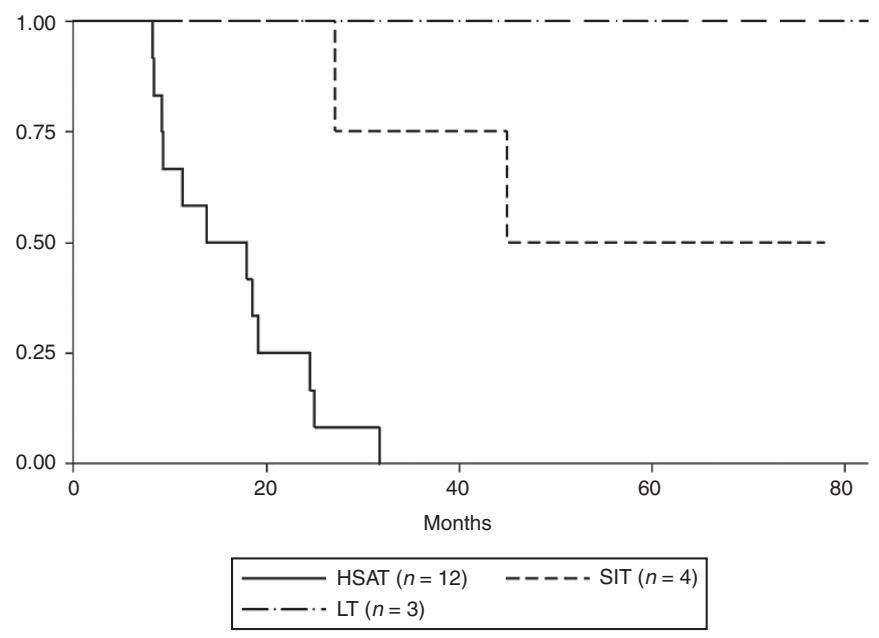

Figure 2 Kaplan-Meier curves* for (a) overall survival and (b) ventilator-free survival for groups with high sustained antibody titers (HSAT), sustained intermediate titers (SIT), and low titers (LT). (a) Overall survival for HSAT, SIT, and LT groups. (b) Invasive ventilator-free survival for HSAT, SIT, and LT groups. *Patient 10 was successfully rescued using immune tolerance induction (ITI) at 86 weeks and was excluded from these calculations. 
remaining five patients (25\%) are alive and invasive ventilator free. Among the patients who died during the study period $(n=$ 14), the median age at death was 32.5 months (range, 14.7-50.2 months) (Figure $2 \mathbf{b}$ ). For the HSAT group, the median age at death was also 32.5 months (range, 14.7-50.2 months; $n=12$ ), and was 13.8 months for patients dependent on a ventilator (range, 8.2-33.6 months; $n=11$ ), similar to previously published data. ${ }^{5}$ Only one patient from the HSAT group is currently alive; this patient received ITI treatment in the entrenched setting. ${ }^{10}$

\section{Left ventricular mass index}

In this study, all groups had an elevated LVMI at baseline compared with the normal upper limit of $64 \mathrm{~g} / \mathrm{m}^{2}$ for infants without Pompe disease. ${ }^{16}$ The median LVMI for the HSAT group was $180.5 \mathrm{~g} / \mathrm{m}^{2}$ at baseline (range, $89.0-253.0 \mathrm{~g} / \mathrm{m}^{2} ; n=10$ ). A net decrease was observed at 26 weeks of ERT (median, $95.0 \mathrm{~g} / \mathrm{m}^{2}$; range, $56.7-269.0 \mathrm{~g} / \mathrm{m}^{2} ; n=11$ ), followed by a net increase at 52 weeks of ERT (median, $124.8 \mathrm{~g} / \mathrm{m}^{2}$; range, $63.3-178.0 \mathrm{~g} / \mathrm{m}^{2}$; $n=8$ ). For the HSAT group, the median LVMI continued to increase after week 52 of ERT ( $n=3$; data not shown). Patient 18 of the LT group had LVMI values of 128.3 and $50.8 \mathrm{~g} / \mathrm{m}^{2}$ at baseline and week 111, respectively, and did not demonstrate a worsening in LVMI after week 52, as was seen in the HSAT group. Patient 20 in the LT group had LVMI values of 68, 65, and 58g/ $\mathrm{m}^{2}$ at baseline, week 30 , and week 60 , respectively. One patient with SIT (patient 15) had LVMI measurements similar to those in the HSAT group $\left(203 \mathrm{~g} / \mathrm{m}^{2}\right.$ at baseline, $138 \mathrm{~g} / \mathrm{m}^{2}$ at week 26 , and $200 \mathrm{~g} / \mathrm{m}^{2}$ at week 52), whereas another SIT patient's (patient 16) baseline LVMI of $141.5 \mathrm{~g} / \mathrm{m}^{2}$ normalized after 9 months of treatment, consistent with those in the LT group.

\section{DISCUSSION}

Similar to what has been previously noted, $\sim 32 \%$ of our IPD cohort was determined to be $\mathrm{CN}-\mathrm{a}$ clinical factor predicting reduced survival and poor clinical outcomes compared with CP patients, ${ }^{5}$ specifically related to the development of HSAT in response to rhGAA. ${ }^{6}$ The use of ITI protocols has changed the natural history of CN IPD, with a significant difference in survival compared with CN IPD treated with ERT monotherapy. ${ }^{9}$ Interestingly, only three $\mathrm{CN}$ cases have been reported in the literature to date that have not developed a significant immune response against rhGAA without the use of ITI, ${ }^{11,12}$ which suggests the presence of other modifying or protective factors influencing treatment outcomes in these cases.

Given the advent of newborn screening for Pompe disease, we sought to determine whether early initiation of ERT prevents the development of an immune response. As previously noted, despite early initiation of treatment, the majority of CN patients with IPD developed sustained antibodies against rhGAA, many of whom mounted HSAT associated with subsequent clinical decline and ultimately death, including some patients who started ERT before 1 month of age. ${ }^{17}$ Therefore, in contrast to previous claims, ${ }^{12}$ early initiation of ERT does not protect against the development of an immune response against rhGAA. ${ }^{18}$
While two CN patients with early initiation of ERT did not develop a clinically significant immune response, both carried splice-site mutations. As such, the patients may have been exposed to GAA epitopes similar to rhGAA if these mutations allowed for residual GAA production, yet at a level below detection by traditional western blot analysis. A closer look at the types of GAA mutations in our cohort showed that most of the patients with HSAT had at least one nonsense GAA mutation. By contrast, $\mathrm{CN}$ patients in the LT group exclusively carried splicing or frameshift GAA mutations, none of which were nonsense mutations.

Leaky splice-site mutations or premature stop codons that occur toward the end of the GAA coding region may possibly result in some residual enzyme activity, which in turn helps prevent the development of an immune response to ERT. The current understanding of CRIM status is based on results from western blots; however, without correlation of GAA mutations, western blot may not provide the entire picture. The use of additional technologies, such as an "omics" approach, may be warranted to identify potential modifying or protective factors and reach a more complete understanding of immune responses in IPD. In addition, the definitive role of various GAA mutations in protein expression as well as the possibility of variable tissue expression needs further exploration.

In conclusion, few $\mathrm{CN}$ patients have been reported with no immune response; however, these patients have unique genotypes, frequently with splicing mutations, whose various degrees of immunogenicity needs to be further explored. This study confirms that $\mathrm{CN}$ status yields an overall poor clinical response; the majority of $\mathrm{CN}$ patients with IPD treated with ERT monotherapy develop an immune response and experience subsequent clinical decline or death. On the contrary, development of anti-rhGAA antibody titers seems to be dictated by the presence of GAA mutations known to severely affect protein expression; thus the results of western blot analysis should be correlated with GAA genotype for the confirmation of CRIM status in IPD. While early diagnosis and treatment are significant in improving overall clinical outcomes in IPD, early diagnosis and initiation of treatment alone do not prevent the development of antibodies against rhGAA. ITI in the naive setting has proven to be successful in preventing the development of antibody titers in CN IPD, which validates the use of ITI therapies in the ERT-naive setting. Because immunological responses accompanied by clinical decline poses a significant risk for the CN IPD population, implementation of ITI in the naive setting should be considered standard of care in treating these patients at risk of developing HSAT. Further investigation surrounding immune responses and the management of IPD, irrespective of CRIM status, is required and warrants attention, particularly in the setting of newborn screening for Pompe disease.

\section{SUPPLEMENTARY MATERIAL}

Supplementary material is linked to the online version of the paper at http://www.nature.com/gim 


\section{ACKNOWLEDGMENTS}

This research was supported by a grant from the Genzyme Corporation, a Sanofi Company (Cambridge, MA), and in part by the Lysosomal Disease Network, a part of National Institutes of Health (NIH) Rare Diseases Clinical Research Network (RDCRN). The Lysosomal Disease Network (U54NS065768) is supported through collaboration between the NIH Office of Rare Diseases Research (ORDR) at the National Center for Advancing Translational Science (NCATS), the National Institute of Neurological Disorders and Stroke (NINDS), and the National Institute of Diabetes and Digestive and Kidney Diseases (NIDDK). The authors are grateful to the patients and their families who participated in this study. They thank Jian Dai for providing western blot results and the local physicians and clinical care team for their collaboration, including Stephanie DeArmey, David Viskochil, Jennifer Propst, Virginia Proud, and Mary-Alice Abbott.

The content is solely the responsibility of the authors and does not necessarily represent the official views of the National Institutes of Health. The funders had no role in study design, data collection and analysis, decision to publish, or preparation of the manuscript.

\section{DISCLOSURE}

P.S.K. and D.S.B. report receiving research and grant support from Genzyme. P.S.K. also receives honoraria and consulting fees from Genzyme and is a member of the Pompe Disease and Gaucher Disease Registry Advisory Boards. C.E., K.B., and A.T.-S. have received honoraria from Genzyme. Duke University and the inventors of the method of treatment and precursors of the cell lines used to generate the enzyme (rhGAA) used commercially have received royalties pursuant to the University's policy on inventions, patents, and technology transfer. This potential conflict for Duke University has been resolved through monetization. The other authors declare no conflict of interest.

\section{REFERENCES}

1. Nicolino M, Byrne B, Wraith JE, et al. Clinical outcomes after long-term treatment with alglucosidase alfa in infants and children with advanced Pompe disease. Genet Med 2009;11:210-219.

2. Kishnani PS, Nicolino M, Voit T, et al. Chinese hamster ovary cell-derived recombinant human acid alpha-glucosidase in infantile-onset Pompe disease. J Pediatr 2006;149:89-97.

3. Kishnani PS, Corzo D, Nicolino M, et al. Recombinant human acid [alpha]glucosidase: major clinical benefits in infantile-onset Pompe disease. Neurology 2007;68:99-109.
4. Kishnani PS, Corzo D, Leslie ND, et al. Early treatment with alglucosidase alpha prolongs long-term survival of infants with Pompe disease. Pediatr Res 2009;66:329-335.

5. Kishnani PS, Goldenberg PC, DeArmey SL, et al. Cross-reactive immunologic material status affects treatment outcomes in Pompe disease infants. Mol Genet Metab 2010;99:26-33.

6. Banugaria SG, Prater SN, Ng YK, et al. The impact of antibodies on clinical outcomes in diseases treated with therapeutic protein: lessons learned from infantile Pompe disease. Genet Med 2011;13:729-736.

7. Mendelsohn NJ, Messinger YH, Rosenberg AS, Kishnani PS. Elimination of antibodies to recombinant enzyme in Pompe's disease. N Engl J Med 2009;360:194-195.

8. Messinger YH, Mendelsohn NJ, Rhead W, et al. Successful immune tolerance induction to enzyme replacement therapy in CRIM-negative infantile Pompe disease. Genet Med 2012;14:135-142.

9. Banugaria SG, Prater SN, Patel TT, et al. Algorithm for the early diagnosis and treatment of patients with cross reactive immunologic material-negative classic infantile pompe disease: a step towards improving the efficacy of ERT. PLoS One 2013;8:e67052.

10. Banugaria SG, Prater SN, McGann JK, et al. Bortezomib in the rapid reduction of high sustained antibody titers in disorders treated with therapeutic protein: lessons learned from Pompe disease. Genet Med 2013;15:123-131.

11. Rohrbach M, Klein A, Köhli-Wiesner A, et al. CRIM-negative infantile Pompe disease: 42-month treatment outcome. J Inherit Metab Dis 2010;33:751-757.

12. Al Khallaf HH, Propst J, Geffrard S, Botha E, Pervaiz MA. CRIM-negative Pompe disease patients with satisfactory clinical outcomes on enzyme replacement therapy. JIMD Rep 2013;9:133-137.

13. Bali DS, Goldstein JL, Banugaria S, et al. Predicting cross-reactive immunological material (CRIM) status in Pompe disease using GAA mutations: lessons learned from 10 years of clinical laboratory testing experience. Am J Med Genet C Semin Med Genet 2012;160C:40-49.

14. Abbott MA, Prater SN, Banugaria SG, et al. Atypical immunologic response in a patient with CRIM-negative Pompe disease. Mol Genet Metab 2011;104: 583-586.

15. Kaplan EL, Meier P. Nonparametric Estimation from Incomplete Observations. J Am Stat Assoc 1958;53:457-481.

16. Vogel M, Staller W, Bühlmeyer K. Left ventricular myocardial mass determined by cross-sectional echocardiography in normal newborns, infants, and children. Pediatr Cardiol 1991;12:143-149.

17. Prater SN, Banugaria SG, Morgan C, Sung CC, Rosenberg AS, Kishnani PS. Letter to the editors: concerning "CRIM-negative Pompe disease patients with satisfactory clinical outcomes on enzyme replacement therapy" by Al Khallaf et al. J Inherit Metab Dis 2014;37:141-143.

18. van Gelder CM, Hoogeveen-Westerveld M, Kroos MA, Plug I, van der Ploeg AT, Reuser AJ. Enzyme therapy and immune response in relation to CRIM status: the Dutch experience in classic infantile Pompe disease. J Inherit Metab Dis 2014; DOI 10.1007/s10545-014-9707-6.

19. Hunley TE, Corzo D, Dudek M, et al. Nephrotic syndrome complicating alphaglucosidase replacement therapy for Pompe disease. Pediatrics 2004;114: e532-e535.

20. Banugaria SG, Patel TT, Mackey J, et al. Persistence of high sustained antibodies to enzyme replacement therapy despite extensive immunomodulatory therapy in an infant with Pompe disease: need for agents to target antibody-secreting plasma cells. Mol Genet Metab 2012;105:677-680. 